EGU21-12716

https://doi.org/10.5194/egusphere-egu21-12716

EGU General Assembly 2021

(c) Author(s) 2021. This work is distributed under

the Creative Commons Attribution 4.0 License.

\title{
Unsupervised Solar Wind Classification Using Wavelet Variational Autoencoders and Self-Organazing Maps
}

\author{
Jorge Amaya, Sara Jamal, and Giovanni Lapenta \\ KU Leuven, CmPA, Mathematics, Leuven, Belgium (jorge.amaya@kuleuven.be)
}

Last year we published an automatic method for the automatic classification of the solar wind [1]. We showed that data transformation and unsupervised clustering can be used to classify observations made by the ACE spacecraft. Two data transformation techniques were used: Kernel Principal Component Analysis (KPCA) and Auto-encoder Neural Networks. After data transformation three clustering techniques were tested: k-means, Bayesian Gaussian Mixtures (BGM), and Self-Organizing Maps (SOM). Although the results were very positive we ran into a few difficulties: a) the data from the ACE mission contains a very small population of observations originated at high latitude coronal holes, b) the measured features contain a high degree of intercorrelation, c) the data distribution is compact in the feature space, and d) the final algorithm produces a single categorical class for a single point in time.

In this work we present an improvement of the model that redresses some of the limitations above. We are still making use of the two main features of our previous work, i.e. the data transformation using auto-encoders and the unsupervised classification using SOM. But in the present work: a) we include the analysis of Ulysses data with observations of the solar wind originated at high latitudes; b) we perform a Factor Analysis to reduce the number of features used as inputs; c) we transform windows of time of the multi-variate time series (instead of instantaneous observations) into scalograms using wavelet transformations; d) we apply the variational version of the auto-encoder [2] to parametrize the scalograms; f) we finally use the SOM to automatically classify the windows of time in different categories.

This method can be adapted to the classification of observations from the Parker Solar Probe and Solar Orbiter missions.

The work presented in this abstract has received funding from the European Union's Horizon 2020 research and innovation programme under grant agreement No. 754304 (DEEP-EST, www.deepprojects.eu), and from the European Union's Horizon 2020 research and innovation programme under grant agreement No 776262 (AIDA, www.aida-space.eu).

[1] Amaya, Jorge, Romain Dupuis, Maria Elena Innocenti, and Giovanni Lapenta. "Visualizing and Interpreting Unsupervised Solar Wind Classifications." arXiv preprint arXiv:2004.13430 (2020).

[2] Kingma, Diederik P., and Max Welling. "Auto-encoding variational bayes." arXiv preprint 
arXiv:1312.6114 (2013). 